\title{
Assessing Participant Feedback to Dental Screenings Provided by Dental Hygiene Students and Follow-up Dental Hygiene Care in New York City
}

Susan H. Davide

CUNY New York City College of Technology, sdavide@citytech.cuny.edu

Margarita Rivera

Farmingdale State College, margarita.rivera@farmingdale.edu

Follow this and additional works at: https://nsuworks.nova.edu/ijahsp

Part of the Dental Hygiene Commons, and the Dental Public Health and Education Commons

\section{Recommended Citation}

Davide SH, Rivera M. Assessing Participant Feedback to Dental Screenings Provided by Dental Hygiene Students and Follow-up Dental Hygiene Care in New York City. The Internet Journal of Allied Health Sciences and Practice. 2021 Jan 01;19(3), Article 8.

This Concept Piece is brought to you for free and open access by the College of Health Care Sciences at NSUWorks. It has been accepted for inclusion in Internet Journal of Allied Health Sciences and Practice by an authorized editor of NSUWorks. For more information, please contact nsuworks@nova.edu. 


\title{
Assessing Participant Feedback to Dental Screenings Provided by Dental Hygiene Students and Follow-up Dental Hygiene Care in New York City
}

\begin{abstract}
Objectives: The purpose of this study was to validate the association between dental screenings and follow-up care to support the level of awareness of dental hygiene services available in the community by increasing access to care and determine patients' knowledge of dental insurance status. Methods: A first-time dental screening was conducted, and complimentary care was provided for those who followed through with dental hygiene care at the clinic. Survey questionnaires were administered to participants following screening and clinic care. Results: Ten participants of the 50 screened received care at the clinic. Less than half of those screened ( $41 \%)$ had a dental visit over a year ago. Most participants were not aware of the low-cost dental services available. Twenty percent $(20 \%)$ of participants did not know if they had dental insurance coverage. Conclusions: Dental screening participants are likely to schedule an appointment for dental services. Raising awareness of low-cost dental care services available in the community by offering screenings at local events may increase access to care.
\end{abstract}

\section{Author Bio(s)}

Susan H. Davide, MSEd, MS, RDH, is an Associate Professor in the department of Dental Hygiene at New York City College of Technology in Brooklyn, N.Y.

Margarita Rivera, MSDH, RDH, is an Assistant Professor in the department of Dental Hygiene at Farmingdale State College in Farmingdale N.Y.

\section{Acknowledgements}

The authors thank Nazrin Akbarova, Dirien Santos and Roseanna Torres, student research assistants, for their active involvement and support. 


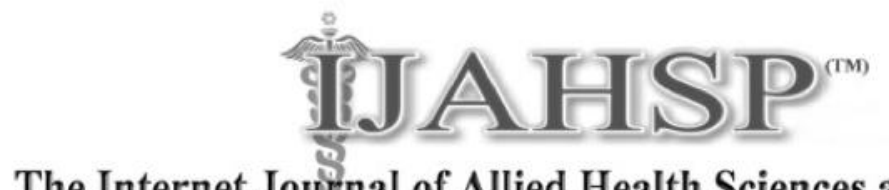

\title{
The Internet Joumnal of Allied Health Sciences and Practice \\ Dedicated to allied health professional practice and education
}

Vol. 19 No. 3 ISSN 1540-580X

\section{Assessing Participant Feedback to Dental Screenings Provided by Dental Hygiene Students and Follow-up Dental Hygiene Care in New York City}

\author{
Susan H. Davide ${ }^{1}$ \\ Margarita Rivera ${ }^{2}$
}

1. CUNY New York City College of Technology

2. Farmingdale State College

United States

\begin{abstract}
Objectives: The purpose of this study was to validate the association between dental screenings and follow-up care to support the level of awareness of dental hygiene services available in the community by increasing access to care and determine patients' knowledge of dental insurance status. Methods: A first-time dental screening was conducted, and complimentary care was provided for those who followed through with dental hygiene care at the clinic. Survey questionnaires were administered to participants following screening and clinic care. Results: Ten participants of the 50 screened received care at the clinic. Less than half of those screened $(41 \%)$ had a dental visit over a year ago. Most participants were not aware of the low-cost dental services available. Twenty percent $(20 \%)$ of participants did not know if they had dental insurance coverage. Conclusions: Dental screening participants are likely to schedule an appointment for dental services. Raising awareness of low-cost dental care services available in the community by offering screenings at local events may increase access to care.
\end{abstract}

Keywords: dental screenings, oral health, dental hygiene care

(C) The Internet Journal of Allied Health Sciences and Practice, 2021 


\section{INTRODUCTION}

Over the past 50 years, the oral health of Americans has made substantial progress as a result of effective prevention and treatment methods. ${ }^{1}$ This includes community water fluoridation and dental sealants, both evidence-based intervention methods to prevent tooth decay. According to the report "Healthy People 2020," oral health is essential to overall health, and access to dental services is vital to promoting and maintaining oral health. ${ }^{1}$ Yet, disparities in access to care remain a continued public health challenge for persons of all age groups. ${ }^{2,3}$ Barriers that affect the ability for individuals to access and/or navigate the oral health care system may be related to low socioeconomic status; shortage of dentists; lack of training in evidence-based oral health guidelines; diminished interdisciplinary collaboration and continuity of care; low oral health literacy; perceptions of need for care; cost; fear of dental procedures; and misconceptions about preventive dental care. ${ }^{4}$

The purpose of this community health initiative was to validate the association between dental screenings and follow-up care to support increasing the level of awareness of dental hygiene services available. The activity consisted of two phases -- a dental screening and a post-screening dental hygiene care appointment. Findings demonstrated the importance of participating in community health fairs to provide oral health screenings in effort to educate the public and provide resource linkage and access to oral health care services.

\section{METHODS}

A first-time dental screening was conducted at a college wellness fair in April of 2018 for two-fold purposes: 1) to screen patients for oral health status and 2) to recruit patients for second year dental hygiene students' clinical care experience. Human subject research exempt status was granted (File \# 2018-1000). The first-round survey included screening, informed consent, instructions, and a questionnaire. To assess receipt of an annual oral examination, participants were asked, "When was your most recent dental visit?" Response options included within the past year; last year; prior to 2017; or unknown/not indicated. Another survey question was, "Do you currently have dental insurance?" Response choices included yes, no, or not sure.

The second-round survey was conducted October 2018 - May 2019, following dental hygiene care. A post-treatment self-rated questionnaire asked: 1) Did you schedule the appointment as a result of the screening event you participated in?; 2) Did you receive relevant information related to your dental care?; 3) Would you return to the clinic for future dental hygiene care?; and 4) Were you pleased with your dental treatment received at our clinic? Completed post-treatment surveys were collected by a student research assistant and placed into a locked depository box located in the clinic. Responses were entered onto a Microsoft Excel spreadsheet to determine data patterns and facilitate reporting results.

\section{RESULTS}

A total of 50 participants ages $25-73$ were screened and completed the first-round survey $(n=50)$. Participants indicated they had a dental visit "a year ago" in 2017 (40\%); did not have any dental visits (22\%); last dental visit was prior to $2017(20 \%)$ and had a dental visit in $2018(16 \%)$. When asked about dental insurance coverage, $65 \%$ reported having insurance; $20 \%$ unsure; $12 \%$ uninsured, and $2 \%$ did not indicate insurance status. Participants were unaware of low-cost services provided by the dental hygiene clinic situated at the local college.

Ten participants followed through with the second phase, by receiving dental hygiene services at the clinic, and completing the second-round survey $(n=10)$. Ninety percent were pleased with the treatment received and would return for future services. One participant was not pleased with the service and indicated the time commitment for the appointment length was the reason. Respondents rated the low cost of services as: excellent $(50 \%)$, good $(10 \%)$, fair $(10 \%)$, and not applicable $(30 \%)$. All ten participants who received services by a dental hygiene student responded "yes" to the survey question, Was the dental hygiene student courteous and professional? Location of services provided was rated as: excellent (80\%), and good (20\%). When asked, Would you recommend our services to your friends and family?, $90 \%$ answered "yes" while $10 \%$ answered "no." Sixty percent stated they received "excellent" oral health information relevant to their dental care, and 40\% reported receiving "good" information.

(C) The Internet Journal of Allied Health Sciences and Practice, 2021 


\section{Most Recent Dental Visit}

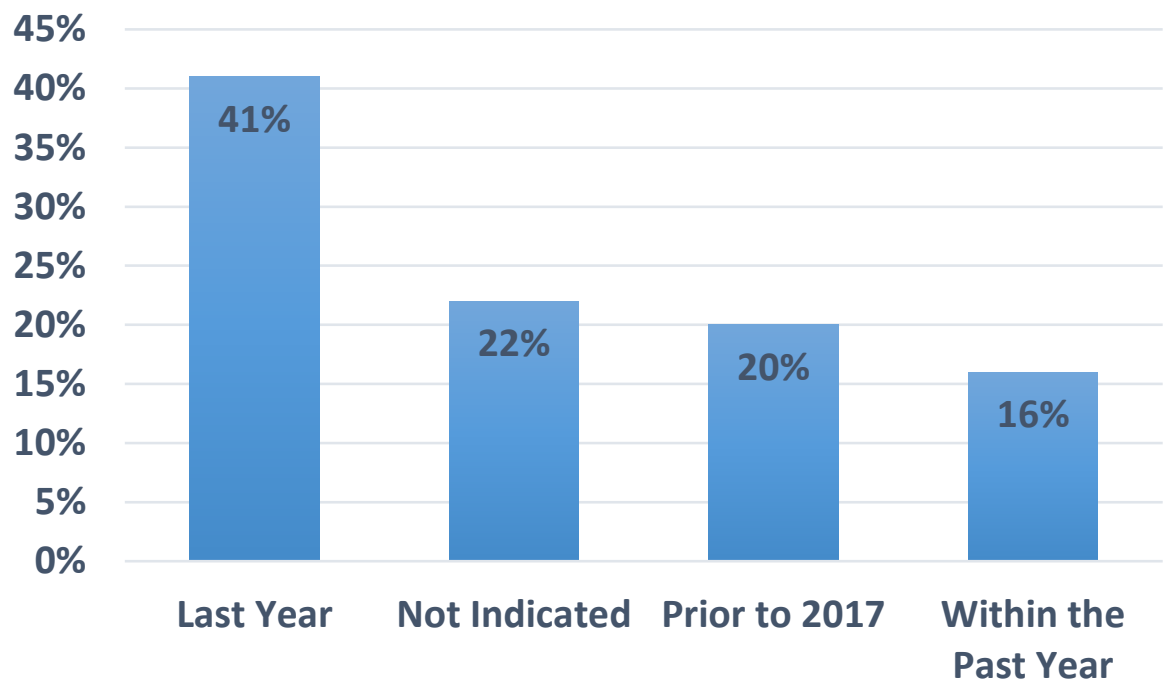

Figure 1. Most recent dental visit

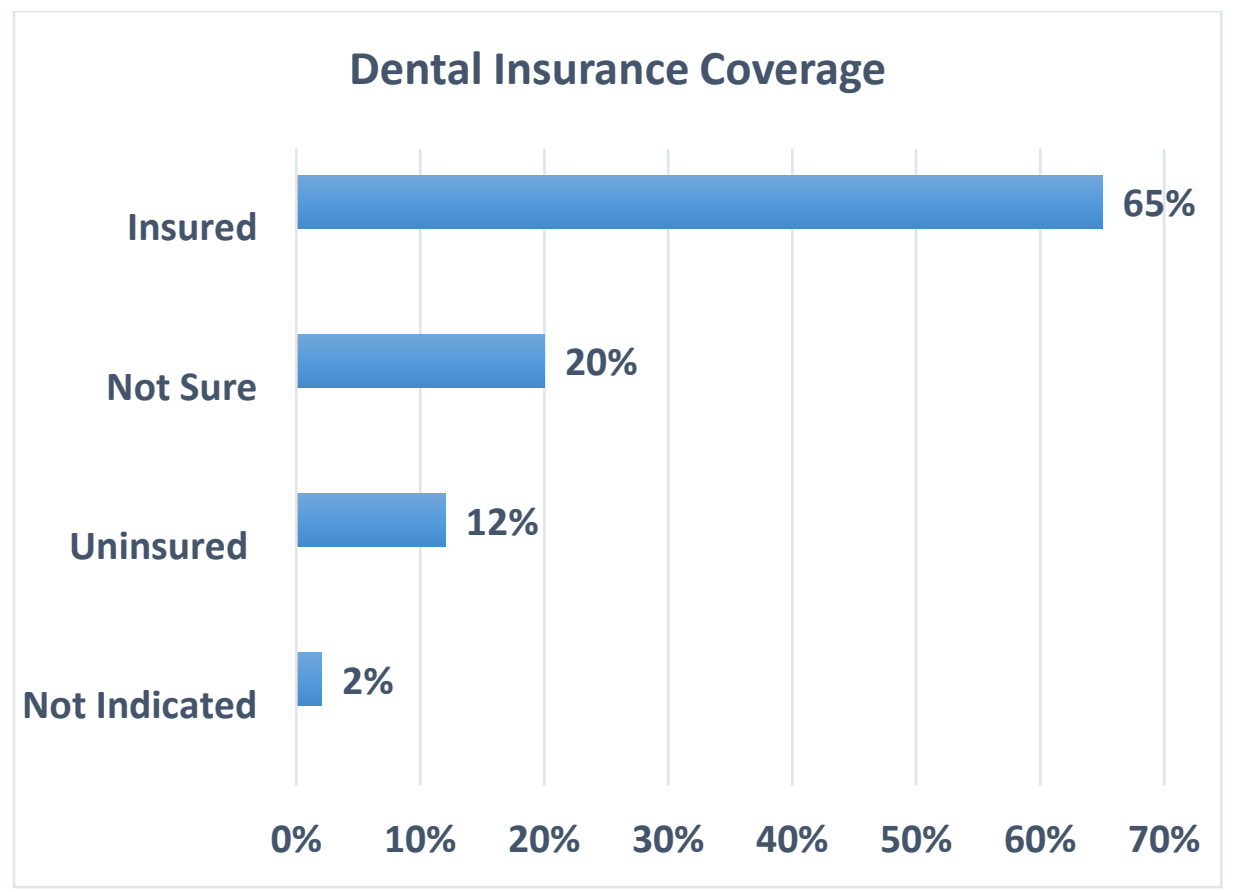

Figure 2. Dental Insurance coverage

\section{DISCUSSION}

Results of this initiative supports the importance of offering dental screenings at local public events to increase awareness of dental hygiene facilities available to the community and its low-cost services to the public. Although no previous studies were found related to this initiative, research indicates there are continuous disparities impacting access to dental care in this country.5,6 The Center for Disease Control and Prevention's Division of Oral Health and the U.S. Department of Health and Human Services both advocate to eliminate oral disparities using strategies, goals, and partnerships. ${ }^{7,8}$ This first-time dental screening event was a partnership 
with a local event. It was noted that by providing participants with linkage to care, they were more likely follow through with the second phase, impacting their oral health and providing preventive services they may not have accessed otherwise.

This community health initiative had several limitations. Dental screening participants were offered a complimentary dental hygiene exam and cleaning as an incentive to schedule an appointment at the clinic for the second phase of the study. The study's second phase was conducted at the dental hygiene clinic in an education institution, where the length of time to receive care requires several appointments approximately 3 hours in length, which may have resulted in reporting bias. The community-based participant sample was noteworthy in number for the dental screening; however, the second phase of participants was relatively low, limiting the post-treatment survey results.

Previously attended health events intended to recruit patients and provide information about the institution's clinical services, and where no screenings were provided, rarely resulted in appointments scheduled. Providing a dental screening presented positive results in patient recruitment and increasing access to care by providing free dental screenings along with incentives for a firsttime complimentary service if participants followed through with their appointment at the institution's clinic. This activity may be a model for other dental and dental hygiene educational programs to promote and attend community events by providing screenings and linkage to care, fulfilling students' needs for patient recruitment and care experiences.

\section{PUBLIC HEALTH IMPLICATIONS}

Oral health is an essential component of overall health. Despite continuous efforts to promote public health awareness of the relationship between oral and systemic diseases, regular dental care is essential. ${ }^{9}$ More than 49 million Americans live in places that are dentally underserved. ${ }^{4}$ The American Dental Association and American Dental Hygienists' Association recommend biannual preventative dental care. This initiative demonstrated when participants receive screenings, they are likely to schedule appointments for services at school dental facilities. Findings also revealed that screened participants visited the local dental hygiene school for services when made aware of this low-cost resource. This initiative also supports raising awareness of the available dental care resources and can aid in increasing access to care for those without dental insurance. Future considerations to investigate should include healthcare navigation for dental insurance perceptions and how partnerships with dental schools can increase access to care. The dental profession has the responsibility to promote oral health for all people, and dental hygiene programs are an integral part of the access to care solution.

\section{ACKNOWLEDEMENTS}

We thank our research assistants: Ms. Nazrin Akbarova, Ms. Dirien Santos, and Ms. Roseanna Torres; former dental hygiene students. We also thank all the study participants for their contribution to the study and Professor Joycelyn Dillon, former chairperson, who valued and supported this research initiative.

\section{REFERENCES}

1. U.S. Department of Health and Human Services. Office of Disease Prevention and Health Promotion (ODPHP).Oral Health. Healthy People 2020 [Internet]. (cited 2020 June 24). Available from: https://www.healthypeople.gov/2020/topics-objectives/topic/oral-health.

2. Brennan Ramirez LK, Baker EA, Metzler M. Promoting health equity: A resource to help communities address social determinants of health. Atlanta: U.S. Department of Health and Human Services, U.S. Centers for Disease Control and Prevention; 2008.

3. U.S. Department of Health and Human Services. National Call to Action to Promote Oral Health. Rockville, MD: U.S. Department of Health and Human Services, Public Health Service, National Institutes of Health, National Institute of Dental and Craniofacial Research. NIH Publication No. 03-5303, Spring 2003. [PMID 21028754]

4. Bersell CH. Access to oral health care: A national crisis and call for reform. J Dent Hyg. 2017;91(1):6-14.

5. Ebersole JL, D'Souza R, Gordon S, Fox CH. Oral health disparities and the future face of America. J of Dent Res. 2012;91(11):997-1002. [PMID 23033549]

6. Henshaw MM, Garcia RI, Weintraub JA. Oral health disparities across the life span. Dent Clin North Am. 2018;62(2):177-193. [PMID 29478452]

7. Department of Health and Human Services, Centers for Disease Control and Prevention (CDC). Oral health: Working to improve oral health for all Americans; at a glance 2016 [Internet]. Atlanta (cited 2020 June 24). Available from: https://www.cdc.gov/chronicdisease/resources/publications/aag/oral-health.htm. 
8. U.S. Department of Health and Human Services Oral Health Coordinating Committee. U.S. Department of Health and Human Services Oral Health Strategic Framework, 2014-2017. Public Health Rep. 2016;131(2):242-257. [PMID: 26957659]

9. Orozco M, Becerra K, Shumulinsky V. Improving access to oral healthcare among low-income older adults: Good oral health can advance health outcomes and quality of life for older adults. Generations. Dec 2019:70-74. 